\title{
Differential Stiffening between the Abdominal and Thoracic Aorta: Effect of Salt Loading in Stroke-Prone Hypertensive Rats
}

\author{
George Lindesay ${ }^{a}$ Yvonnick Bézie $^{b}$ Christophe Ragonnet $^{a}$ \\ Véronique Duchatelle ${ }^{c}$ Chandima Dharmasena ${ }^{b}$ Nicole Villeneuve $^{a}$ \\ Christine Vayssettes-Courchay ${ }^{a}$ \\ ${ }^{a}$ Cardiovascular Discovery Research Unit Suresnes, Servier Research Institute, Suresnes, France; ${ }^{b}$ Department of \\ Pharmacy, Groupe Hospitalier Paris Saint-Joseph, Paris, France; ${ }^{C}$ Department of Pathology, Groupe Hospitalier Paris \\ Saint-Joseph, Paris, France
}

\section{Keywords}

Aortic distensibility · Elastin disarray · Blood pressure ·

Aortic stiffness

\begin{abstract}
Central artery stiffening is recognized as a cardiovascular risk. The effects of hypertension and aging have been shown in human and animal models but the effect of salt is still controversial. We studied the effect of a high-salt diet on aortic stiffness in salt-sensitive spontaneously hypersensitive stroke-prone rats (SHRSP). Distensibility, distension, and $\beta$-stiffness were measured at thoracic and abdominal aortic sites in the same rats, using echotracking recording of the aortic diameter coupled with blood pressure (BP), in SHRSPsalt ( $5 \%$ salted diet, 5 weeks), SHRSP, and normotensive Wistar-Kyoto (WKY) rats. Hemodynamic parameters were measured at BP matched to that of WKY. Histological staining and immunohistochemistry were used for structural analysis. Hemodynamic isobaric parameters in SHRSP did not differ from WKY and only those from the abdominal aorta of SHRSP-salt presented decreased distensibility and increased stiffness compared with WKY and SHRSP. The abdominal and thoracic aortas presented similar thickening, increased fibrosis, and remodeling with no change in colla-
\end{abstract}

gen content. SHRSP-salt presented a specific increased elastin disarray at the abdominal aorta level but a decrease in elastin content in the thoracic aorta. This study demonstrates the pro-stiffening effect of salt in addition to hypertension; it shows that only the abdominal aorta presents a specific pressure-independent stiffening, in which elastin disarray is likely a key mechanism.

(c) 2018 S. Karger AG, Basel

\section{Introduction}

The development of arterial stiffness is an independent risk factor for cardiovascular events and all-cause mortality. It occurs naturally with aging and also with hypertension and metabolic disorders [1-3]. It is well known that age and hypertension each play a role in worsening existing increases in arterial stiffness $[4,5]$. Indeed, increased cyclic (pulsatile) and steady pressure upon the arterial wall leads to further vascular remodeling characterized by a reduction in elastin and an increase in fibrous tissue in the arterial wall. Heretofore, arterial stiffness has been mostly considered as an inevitability of old age or simply a by-product of hypertension, and as such there is a present lack of motivation to find pharmaceutical treat-

\section{KARGER}

(c) 2018 S. Karger AG, Basel

E-Mail karger@karger.com

www.karger.com/jvr
Dr. Christine Vayssettes-Courchay

Cardiovascular Discovery Research Unit, Servier Research Institute

11 rue des Moulineaux

FR-92150 Suresnes (France)

E-Mail christine.courchay@ servier.com 
ments which target stiffness directly. However, some studies suggest that increases in arterial stiffness could precede the development of hypertension in humans [6, 7]. Critical to understanding how to treat this degenerative condition is to first understand the mechanisms through which it manifests. Until now, it has remained difficult to separate the causal effects of blood pressure (BP) elevation on arterial stiffness measurements from those of the long-term changes in mechanical and functional properties of the arterial wall which also lead to alterations in vascular stiffness. It is therefore essential for experimental models to allow for measurements to be made at controlled arterial pressures in order to distinguish the effects of vascular remodeling versus acute pressure-dependent changes in arterial stiffness.

Among the prospective candidates which could induce arterial stiffening is sodium. It has already been shown in humans that dietary sodium is correlated with arterial stiffness independent of BP $[8,9]$. Few data exist for the effects of salt in animal models on arterial stiffness. A recent study showed higher aortic and carotid pulse wave velocity (PWV) independent of BP [10] in salt-sensitive Dahl rats when compared with salt-resistant Dahl rats under a lower-salt diet. This study determined that the origin of the higher stiffness was not due to classic fibroid deposition into the arterial extracellular matrix, but rather a change in the molecular expression of the constituents of the media and endothelium. In this study, as in those in humans, the benefit of a low-salt diet was observed. Nevertheless, the deleterious effects of an increased salt diet which could mimic the salt component of the western diet is still poorly investigated.

Salt loading in spontaneously hypertensive strokeprone rats (SHRSP) increases the incidence of stroke [11, $12]$. The reasons for this are multifactorial, including endothelial [13] and renal dysfunction [14]. Since the endothelium is a primary means of maintaining vascular smooth muscle integrity [15] and renal dysfunction is associated with arterial stiffness [16], it was hypothesized that this model was relevant for analyzing arterial remodeling associated with stiffness.

The goal of the present investigation was to determine whether increases in arterial stiffness could be observed in SHRSP receiving increased dietary salt. As the regional differences in stiffness across the aorta have been poorly investigated and remain a source of controversy, we fully investigated stiffness after salt loading at both abdominal and thoracic sites via local echotracking stiffness measurements $[5,17]$ and subsequently measured the associated alterations in aortic tissue $[18,19]$.

Salt-Induced Aortic Stiffening in Rats

\section{Methods}

\section{Animals}

This study was conducted in accordance with European Community Guidelines for the use of experimental animals and was approved by the ethical committee on Animal Experiments of the Servier Research Institute. All animals were provided by Charles River (L'arbresle, France). Male Wistar-Kyoto (WKY) rats $(n=6)$, SHRSP rats $(n=6)$, and SHRSP rats given a $5 \%$ salt diet for 5 weeks (SHRSP-salt; $n=8$ ) were compared at 20 weeks of age. The animals were housed in a temperature-controlled room $\left(20-21^{\circ} \mathrm{C}\right)$ with a $12 / 12 \mathrm{~h}$ light/dark cycle. The body weight was measured before anesthesia.

\section{Hemodynamic Measurements}

Rats were anaesthetized with an intraperitoneal injection of sodium pentobarbital ( $50 \mathrm{mg} / \mathrm{kg}$, maintained with $5 \mathrm{mg} / \mathrm{kg} / \mathrm{h}$ ). The jugular vein was cannulated for constant administration of anesthetic and the penile vein was cannulated for the administration of other drugs. The trachea was cannulated and ventilation was maintained with a pressure-controlled respirator (Hallowell EMC, TEM) at a frequency of 60-70 cycles/min and a pressure of 9-12 $\mathrm{cm} \mathrm{H}_{2} \mathrm{O}$ to avoid respiratory depression during the experiment and to allow better stability. Body temperature was maintained at $37^{\circ} \mathrm{C}$ with a homeothermic blanket (Harvard) connected to a rectal probe.

A microtip pressure catheter (Millar 1.2F) was inserted into the aorta via the right femoral artery. The BP signal was visualized and analyzed with the Biopac 4.2 Acknowledge acquisition and analysis system (CEROM). The aortic diameter was simultaneously measured with pressure using ultrasound. An ultrasound probe (L10-5, $40 \mathrm{~mm}, 10 \mathrm{MHz}$ ) was placed on the shaved skin on the side of the animal and was manipulated until a clear B-mode image of the thoracic aorta and the intra-arterial catheter was seen. A section of artery adjacent to the catheter was selected and subsequently analyzed in M-mode. Vessel wall tracking technology (Art.Lab, Esaote, Maastricht, The Netherlands) was used to measure the changes in arterial diameter for $6 \mathrm{~s}$ (approx. 30 cardiac cycles). The $\mathrm{BP}$ signal was split and sent to a second computer containing Art. Lab to allow for $\mathrm{BP}$ and diameter synchronization. These data were subsequently analyzed using a specialized Matlab (Mathworks) program which integrates $\mathrm{BP}$ and diameter data and therefore allowed for arterial stiffness measurements. A more detailed description of both data acquisition via Art.Lab [20] and the specific analyses within Matlab [17] have previously been published.

Thoracic measurements were made above the renal artery bifurcation and below the diaphragm, and abdominal aorta measurements were made at the lowest abdominal site. The catheter was inserted first into the thoracic aorta via the right femoral artery and measurements were made in the thoracic aorta at baseline BP. Then the catheter was withdrawn and placed in the abdominal aorta just above the iliac bifurcation. The ultrasound was also moved to obtain an image of the catheter within the abdominal aorta. For the SHRSP and SHRSP-salt groups, after a second series of baseline measurements were taken within the abdominal aorta, $\mathrm{BP}$ was reduced using an injection of clonidine $(3 \mu \mathrm{g} / \mathrm{kg}$ i.v.) to achieve the same baseline $\mathrm{BP}$ as the normotensive rats (approx. 130 $\mathrm{mm} \mathrm{Hg}$ ). Following these measurements, the catheter and ultrasound probe were repositioned into and above the thoracic aorta to obtain measurements at the baseline BP of the normotensive 


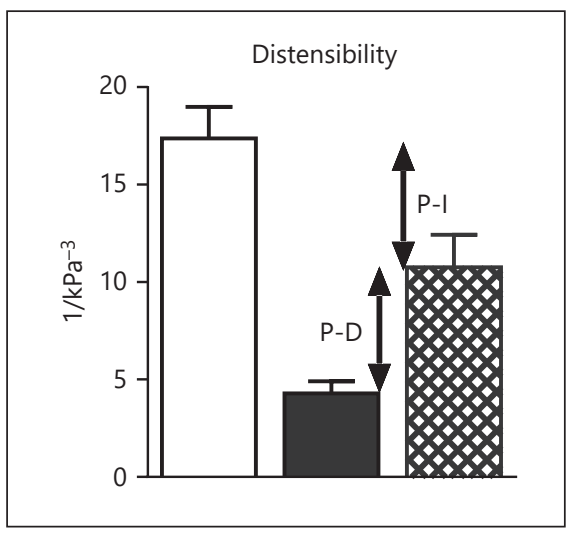

Fig. 1. Illustration of the way to determine pressure-dependent (PD) and pressure-independent (P-I) changes in arterial distensibility. The filled bar shows the decreased distensibility in the pathological model compared with the normotensive rat (white bar), whilst the hatched bar shows distensibility at a BP matched to the white bar: its recovered part is P-D stiffening and the remaining reduction is the P-I stiffening.

rats. The changes induced by the reduction in BP represent the pressure-dependent stiffness and the difference remaining versus normotensive animals after BP reduction represents the pressureindependent stiffening (Fig. 1 shows an example with distensibility).

The parameters automatically calculated to determine the dynamic properties of the aortic wall were as follows: mean diameter $(D)$ and diastolic diameter $(\mathrm{d} D)$; mean distension (in $\mu \mathrm{m})$; compliance $(\Delta A / \Delta \mathrm{P})$ in $\mathrm{mm}^{2} / \mathrm{kPa}$, where $A$ is the transsectional area of the vessel calculated from the diameter, and $\mathrm{P}$ is pressure; distensibility $(\triangle A / \triangle \mathrm{P} \mathrm{X} A)$ in $1 / \mathrm{kPa}$, and beta stiffness index $[(\mathrm{d} D \ln [\mathrm{SAP} /$ $\mathrm{DAP}]) /(\mathrm{s} D-\mathrm{d} D)]$, where $\mathrm{s} D$ is systolic diameter, SAP is systolic arterial pressure, and DAP is diastolic arterial pressure. Aortic distension was expressed in percent versus diastolic diameter $(\triangle D$ $\mathrm{X} 100 / \mathrm{d} D)$. The local PWV is calculated from $\sqrt{ }(\mathrm{A} / \Delta \mathrm{P} / \rho \Delta \mathrm{A})$, where $\rho$ is blood viscosity.

Additionally, as previously described [17], we analyzed the pressure wave and diameter distension wave by recording both signals at $980 \mathrm{~Hz}$ (1.02-ms intervals) and averaging them over around 30 cardiac cycles. Pulse pressure and the distension wave transformed in percent/diastolic diameter were then quantified by the area under the curve (AUCd and AUCpp, respectively) corrected by $1 /$ heart rate to avoid a heart cycle duration influence. The pressure waves and distension waves as well as the AUC/ms obtained for each rat could then be averaged within groups as is usually done for the more standard parameters. A distensibility index was calculated as the ratio of these AUCs, AUCd/AUCpp, and a compliance index was also added as (distension/diastolic diameter)/(pulse pressure/diastolic pressure).

\section{Samples for ex vivo Experiments}

At the end of the experiments the rats were euthanized via an intravenous (i.v.) lethal dose of sodium pentobarbital, and the naso-rectal lengths of the rats were measured. The left ventricle and left kidney were weighed. The left ventricle and left kidney weight were normalized to their ratio to naso-rectal length $(\mathrm{mg} / \mathrm{cm})$. Thoracic and abdominal aortas were cleaned and stored in $4 \%$ formaldehyde.

\section{Determination of Arterial Structure and Composition}

The arterial structure was determined and quantified in $4 \%$ formaldehyde-fixed thoracic and abdominal aortas extracted from the rats used for hemodynamic measurements. For that purpose, an approximately 5-mm-long piece, corresponding to the site already used for both thoracic and abdominal ultrasound measurements, was cut and embedded in paraffin. The tissue was then extracted from individual paraffin blocks and inserted into a preformed paraffin recipient block (Tissue-Tek Quick-Ray System, Sakura Finetek, Villeneuve d'Ascq, France). The finished block was then cut into 4 - $\mu$ m-thick sections and mounted on Superfrost plus slides and subjected to independent tests.

The medial cross-sectional area (MCSA) and scleroprotein quantifications were performed by morphological analysis after Sirius red and a 3-color staining protocol (Masson's trichrome) for collagen [19,21], and after Verhoeff's van Gieson staining for elastin [22]. The disarray of elastin networks in the medial layer was determined by loss of parallel orientation and increased dispersion and rupture of fibers. It was graded with a scale of 3 levels. Level 1 reflected almost all elastin fibers distributed and aligned in parallel throughout the vascular wall. Level 2 was graded for samples with elastin fibers distorted and level 3 for samples with at last half of the elastin fibers distorted or disrupted. For each rat, 3 different microscopic fields were analyzed at $40 \times$ magnification.

For immunohistochemical analyses of cell-matrix interactions, a fibronectin polyclonal antibody (ab2040, Millipore) was used. Integrin accumulation was quantified with $\alpha_{1}$-integrin (ab106267, Abcam), $\alpha_{5}$-integrin (ab1928, Millipore), and $\alpha_{\mathrm{v}}$-integrin (ab1930, Millipore) antibodies. This analysis was completed with an antiFAK antibody (ab40794, Abcam), which recognized focal adhesion kinase (FAK). To complete the description of the aorta composition, MMP2, a ubiquitous metalloproteinase that is involved in the remodeling of the vasculature, was also quantified (antiMMP2 antibody; ab37150, Abcam).

Immunohistochemistry was performed on $4-\mu \mathrm{m}$ sections [18, $19,23]$. Heat-mediated antigen retrieval was performed in EDTA buffer $\mathrm{pH} 9$ in a water bath for $30 \mathrm{~min}$. Immunostaining was performed on a Dako autostainer using a peroxidase-labeled polymerbased detection system (Envision plus, Dako) and diaminobenzidine as a chromogen. No specific staining was observed when the primary antibody was omitted from the protocol (negative control). The distribution and quantification of staining were determined by computer-directed color analysis performed with the noncommercial image processing software Mesurin ${ }^{\circledR}$. For each aortic section analyzed, 3 different microscopic fields were analyzed at 20× magnification [24]. Wall stress was measured using the formula: mean blood pressure (MBP) after clonidine administration $\times$ internal diameter $/ 2 \times$ thickness.

\section{Statistical Analysis}

All data were expressed as the mean \pm the standard error of the mean (SEM). The coefficient of variation measurement (\%) was calculated. Then each hemodynamic parameter was analyzed with a one-way ANOVA of raw data followed by a Tukey post hoc comparison first at basal BP and again at matched BPs across the 3 groups. The paired Student $t$ test was performed to compare tho- 
Table 1. Thoracic aorta hemodynamics, diameter, and stiffness measurements

\begin{tabular}{|c|c|c|c|c|c|}
\hline Mean AP, mm Hg & $130 \pm 1$ & $200 \pm 5^{*}$ & $128 \pm 2$ & $214 \pm 3 *, \#$ & $124 \pm 1^{*}$ \\
\hline Systolic AP, mm Hg & $154 \pm 2$ & $237 \pm 7^{*}$ & $151 \pm 2$ & $251 \pm 4^{*, \#}$ & $149 \pm 1$ \\
\hline Diastolic AP, mm Hg & $111 \pm 2$ & $169 \pm 5^{*}$ & $108 \pm 2$ & $186 \pm 2^{*, \#}$ & $105 \pm 2$ \\
\hline Heart rate, bats/min & $350 \pm 17$ & $381 \pm 4$ & $330 \pm 30$ & $396 \pm 9 *$ & $350 \pm 15$ \\
\hline Mean diameter, $\mu \mathrm{m}$ & $2,241 \pm 27$ & $2,517 \pm 47^{*}$ & $2,376 \pm 48^{*}$ & $2,748 \pm 43^{*, \#}$ & $2,478 \pm 51^{*}$ \\
\hline Diastolic diameter, $\mu \mathrm{m}$ & $2,092 \pm 26$ & $2,376 \pm 58^{*}$ & $2,229 \pm 54^{*}$ & $2,636 \pm 53^{*, \#}$ & $2,354 \pm 48^{*}$ \\
\hline Aortic distension, $\mu \mathrm{m}$ & $192 \pm 12$ & $124 \pm 7$ & $211 \pm 17$ & $88 \pm 7$ & $197 \pm 14$ \\
\hline Aortic distension, $\%$ & $9.2 \pm 0.6$ & $5.2 \pm 0.2^{*}$ & $9.5 \pm 1.0$ & $3.3 \pm 0.3^{*, \#}$ & $8.4 \pm 0.6$ \\
\hline Compliance, $\mathrm{mm}^{2} / \mathrm{kPa} \times 10^{-3}$ & $122.1 \pm 5.6$ & $55.8 \pm 6.2 *$ & $141.0 \pm 7.8$ & $44.8 \pm 4.1$ & $133.3 \pm 64$ \\
\hline Distension, $\mathrm{AUCd} / \mathrm{ms} \times 10^{-1}$ & $4.9 \pm 0.4$ & $2.8 \pm 0.1^{*}$ & $5.0 \pm 0.5$ & $1.7 \pm 0.1^{*, \#}$ & $4.2 \pm 0.4$ \\
\hline Distensibility index $\times 10^{-2}(\mathrm{AUCd} / \mathrm{AUCpp})$ & $25.1 \pm 1.1$ & $9.4 \pm 0.5$ & $25.3 \pm 2.4$ & $6.1 \pm 0.5$ & $22.0 \pm 0.9$ \\
\hline Compliance index & $24.1 \pm 0.6$ & $13.2 \pm 0.7^{*}$ & $24.0 \pm 2$ & $9.8 \pm 0.8^{*, \#}$ & $20.4 \pm 0.9$ \\
\hline$n$ & 6 & 6 & 6 & 8 & 8 \\
\hline
\end{tabular}

Parameters were measured and calculated via the echotracking at the thoracic aorta. Results are expressed as the mean \pm SEM. AP, arterial pressure; PWV, pulse wave velocity. ${ }^{*}$ Significantly different $(p<0.05)$ compared to WKY; ${ }^{*}$ significantly different $(p<0.05)$ compared to SHRSP.

racic and abdominal values, as well as the effect of clonidine in hypertensive rats. For immunochemistry analysis, data are expressed as means \pm SEM. One-way ANOVA followed by a Fisher PLSD (protected least significant difference) was used to assess the significance of the results. Differences were considered significant at values of $p<0.05$.

\section{Results}

\section{Basal and Hemodynamic Data}

The body weights of SHRSP and WKY rats did not differ and that of SHRSP-salt rats was slightly lower, significantly when compared to WKY (WKY $=340 \pm 7 \mathrm{~g}$, SHRSP $=318 \pm 3 \mathrm{~g}$, SHRSP-salt $=311 \pm 6 \mathrm{~g}$ ). Left ventricle and left kidney weights corrected for naso-anal length were higher in SHRSP compared to WKY, and this difference was increased by salt, indicating a hypertrophy of both organs induced by hypertension and increased by the addition of salt in hypertensive rats. Left ventricle values were $38.9 \pm 1.9,44.0 \pm 0.8$, and $49.5 \pm 0.8 \mathrm{mg} / \mathrm{cm}$ in the WKY, SHRSP, and SRHSP-salt groups, and the kidneys were $43.1 \pm 1.8,55.0 \pm 0.9$, and $65.0 \pm 1.9 \mathrm{mg} / \mathrm{cm}$, respectively. Basal values of $\mathrm{MBP}$ in anesthetized rats were greatly increased in SHRSP and SHRSP-salt rats compared to WKY animals. Clonidine i.v. push $(3 \mu \mathrm{g} / \mathrm{kg})$ reduced MBP significantly in both the SHRSP and SHRSP-salt groups, reaching similar values to those measured in WKY rats (Tables 1,2).

As expected, the in vivo diameter in the thoracic aorta was higher than in the abdominal aorta, 2,241 \pm 27 and $1,434 \pm 15 \mu \mathrm{m}$, respectively, in WKY rats. This difference was also seen in the other groups at both basal and reduced BPs (Table 2, 3). Clonidine did not significantly modify the diameter of the abdominal aorta and slightly reduced that of the thoracic aorta.

\section{Aortic Distensibility and Stiffness}

The thoracic aorta was less stiff compared to that of the abdominal aorta in all 3 groups (values are shown in Tables 1 and 2). At resting BP, aortic stiffness was higher in hypertensive animals compared to the normotensive animals, as shown by the higher $\beta$-stiffness index and PWV, and lower distensibility and distension waveform in both the thoracic (Table 1) and the abdominal aorta (Table 2). 
Table 2. Abdominal aorta hemodynamics, diameter, and stiffness measurements

\begin{tabular}{|c|c|c|c|c|c|}
\hline Abdominal aorta & WKY & SHRSP & $\begin{array}{l}\text { SHRSP + } \\
\text { clonidine }\end{array}$ & SHRSP-salt & $\begin{array}{l}\text { SHRSP-salt + } \\
\text { clonidine }\end{array}$ \\
\hline Mean AP, mm Hg & $129 \pm 2$ & $192 \pm 6^{*}$ & $128 \pm 3$ & $200 \pm 5^{*}$ & $129 \pm 1$ \\
\hline Systolic AP, mm Hg & $156 \pm 1$ & $226 \pm 9^{*}$ & $155 \pm 3$ & $234 \pm 5^{*}$ & $156 \pm 3$ \\
\hline Diastolic AP, mm Hg & $109 \pm 2$ & $163 \pm 5^{*}$ & $107 \pm 3$ & $174 \pm 4^{*}$ & $110 \pm 1$ \\
\hline Heart rate, beats/min & $348 \pm 16$ & $375 \pm 7$ & $337 \pm 9$ & $399 \pm 8^{*}$ & $359 \pm 17$ \\
\hline Mean diameter, $\mu \mathrm{m}$ & $1,434 \pm 16$ & $1,369 \pm 49$ & $1,307 \pm 47$ & $1,484 \pm 64$ & $1,457 \pm 50$ \\
\hline Diastolic diameter, $\mu \mathrm{m}$ & $1,344 \pm 19$ & $1,288 \pm 66$ & $1,228 \pm 47$ & $1,424 \pm 68$ & $1,403 \pm 47^{\#}$ \\
\hline Aortic distension, $\mu \mathrm{m}$ & $102 \pm 5$ & $45 \pm 4$ & $78 \pm 6$ & $34 \pm 4$ & $64 \pm 4$ \\
\hline Aortic distension, $\%$ & $7.6 \pm 0.4$ & $3.5 \pm 0.3^{*}$ & $6.4 \pm 0.5$ & $2.5 \pm 0.3^{*, \#}$ & $4.5 \pm 0.3^{*, \#}$ \\
\hline Compliance, $\mathrm{mm}^{2} / \mathrm{kPa} \times 10^{-3}$ & $37.4 \pm 1.2$ & $11.6 \pm 1.5^{*}$ & $24.7 \pm 1.7^{*}$ & $10.4 \pm 1.4^{*}$ & $23.8 \pm 1.4^{*}$ \\
\hline Distension, $\mathrm{AUCd} / \mathrm{ms} \times 10^{-1}$ & $3.9 \pm 0.2$ & $1.8 \pm 0.1^{*}$ & $3.2 \pm 0.2$ & $1.2 \pm 0.1^{*, \#}$ & $2.1 \pm 0.1$ \\
\hline Distensibility index $\times 10^{-2}(\mathrm{AUCd} / \mathrm{AUCpp})$ & $19.1 \pm 0.8$ & $6.3 \pm 0.4^{*}$ & $15.3 \pm 1.1^{*}$ & $4.9 \pm 0.6^{*}$ & $12.0 \pm 08^{*}$ \\
\hline Compliance index & $17.8 \pm 0.6$ & $8.9 \pm 0.6^{*}$ & $14.0 \pm 0.6^{*}$ & $7.4 \pm 0.8^{*}$ & $11.1 \pm 0.8^{*, \#}$ \\
\hline$n$ & 6 & 6 & 6 & 8 & 7 \\
\hline
\end{tabular}

Parameters measured and calculated via the echotracking at the abdominal aorta. Results are expressed as mean \pm SEM. AP, arterial pressure; PWV, pulse wave velocity. * Significantly different $(p<0.05)$ compared to WKY; ${ }^{*}$ significantly different $(p<0.05)$ compared to SHRSP.

For SHRSP rats, salt-induced changes in arterial stiffness were difficult to evaluate at resting BP.

Under isobaric conditions following the bolus of i.v. clonidine, there was no longer a difference in aortic stiffness between WKY, SHRSP, and SHRSP-salt at the thoracic level, indicating the passive contribution of $\mathrm{BP}$ upon arterial stiffness at resting BP in SHRSP and SHRSP-salt (Table 1 ). In contrast, the $\beta$-stiffness index and local PWV recovered and distensibility remained slightly lower in the SHRSP animals at the abdominal aorta level with respect to the WKY group. Furthermore, in the SHRSP-salt group, all the parameters remained altered after BP reduction compared to both the WKY and the SHRSP rats (Table 2). Since these measurements occurred at an equal MBP, the increased stiffness was not influenced by distending pressure.

\section{Distension and BP Waves}

Pulse pressure and pulse pressure waves measured as the AUC/ms were higher in SHRSP and SHRSP-salt compared with WKY rats, with no difference between hypertensive groups at both thoracic and abdominal levels. Fol- lowing BP reduction with clonidine, there were no measured differences in pulse pressure or AUC in any of the groups. In contrast, the distension and distension wave measured as AUC/ms were greatly reduced in SHRSP and more reduced in SHRSP-salt rats at both thoracic and abdominal levels. After clonidine, only distension and distension waves of SHRSP-salt abdominal aorta remained reduced versus WKY (Tables 1, 2; Fig. 2, 3). Figure 3 shows the distension/pressure relationship as a distension-pressure loop throughout the cardiac cycle, when BP in hypertensive rats was lowered to match that of the normotensive rats. The thoracic aorta isobaric slopes and areas of the distension-pressure loop are similar in the 3 groups. At the abdominal level the distension-pressure loop in SHR-salt exhibits a lower slope and area, clearly indicating a pressure-independent stiffening.

\section{Arterial Structure and Composition}

The immunohistochemical characteristics of the thoracic and abdominal aortic structures are presented in Table 3. The MCSA and media thickness increased in SHRSP rats at both sites compared with WKY rats. There 
Table 3. Aortic structure and composition

\begin{tabular}{|c|c|c|c|c|c|c|}
\hline & \multicolumn{3}{|c|}{ Thoracic aortic site } & \multicolumn{3}{|c|}{ Abdominal aortic site } \\
\hline & WKY & SHRSP & SHRSP-salt & WKY & SHRSP & SHRSP-salt \\
\hline Thickness, $\mu \mathrm{m}$ & $90 \pm 1.5$ & $111 \pm 4.0^{*}$ & $129 \pm 5.7^{*, \#}$ & $92 \pm 3.3$ & $106 \pm 3.4^{*}$ & $119 \pm 3.7^{*, \#}$ \\
\hline Thickness/lumen & $7.3 \pm 0.2$ & $7.8 \pm 0.3$ & $8.5 \pm 0.2$ & $10.6 \pm 0.5$ & $13.2 \pm 0.4^{*}$ & $13.4 \pm 0.5^{*}$ \\
\hline MCSA, AU & $1,200 \pm 50$ & $1,647 \pm 41^{*}$ & $1,958 \pm 38^{*, \#}$ & $806 \pm 59$ & $903 \pm 47$ & $1,100 \pm 66^{*, \#}$ \\
\hline MCSA/BW, mg/AU & $3.54 \pm 0.14$ & $5.19 \pm 0.16^{*}$ & $6.33 \pm 0.21^{*, \#}$ & $2.37 \pm 0.15$ & $2.84 \pm 0.13^{*}$ & $3.56 \pm 0.23^{*}$ \\
\hline Media thickness, $\mathrm{AU}$ & $5.61 \pm 0.10$ & $6.46 \pm 0.12 *$ & $7.31 \pm 0.13^{*, \#}$ & $5.36 \pm 0.32$ & $6.26 \pm 0.27^{*}$ & $7.06 \pm 0.21^{*, \#}$ \\
\hline Elastic lamellae, $n$ & $7.5 \pm 0.2$ & $8.8 \pm 0.4$ & $8.3 \pm 04$ & $5.83 \pm 0.31$ & $6.33 \pm 0.42$ & $7.13 \pm 0.30^{*}$ \\
\hline Interlamellar space, $\mathrm{AU}$ & $0.75 \pm 0.02$ & $0.74 \pm 0.04$ & $0.89 \pm 0.06$ & $0.93 \pm 0.07$ & $1.01 \pm 0.08$ & $1.03 \pm 0.07$ \\
\hline \multicolumn{7}{|l|}{ Colored aortic wall } \\
\hline VSMC nucleus & $7.8 \pm 0.5$ & $9.6 \pm 0.6^{*}$ & $9.7 \pm 0.5^{*}$ & $7.17 \pm 0.47$ & $9.60 \pm 0.68^{*}$ & $9.4 \pm 0.76^{*}$ \\
\hline Collagen density, \% & $24.5 \pm 1.1$ & $24.1 \pm 1.7$ & $26.3 \pm 0.7$ & $22.4 \pm 1.1$ & $22.6 \pm 1.0$ & $23.0 \pm 0.8$ \\
\hline Elastin density, \% & $21.4 \pm 0.9$ & $21.3 \pm 0.5$ & $18.3 \pm 0.6^{*, \#}$ & $15.4 \pm 0.7$ & $13.7 \pm 0.7$ & $15.07 \pm 0.7$ \\
\hline Elastin/collagen & $0.89 \pm 0.07$ & $0.88 \pm 0.05$ & $0.70 \pm 0.03^{*, \#}$ & $0.70 \pm 0.05$ & $0.62 \pm 0.05$ & $0.67 \pm 0.04$ \\
\hline \multicolumn{7}{|l|}{ Stained aortic wall, \% } \\
\hline Fibronectin & $7.32 \pm 0.27$ & $7.42 \pm 0.35$ & $10.53 \pm 0.49^{*, \#}$ & $5.87 \pm 0.42$ & $6.61 \pm 0.27$ & $8.86 \pm 0.40^{*, \#}$ \\
\hline$a_{1}$-integrin & $2.22 \pm 0.33$ & $2.68 \pm 0.57$ & $2.62 \pm 0.43$ & $1.46 \pm 0.20$ & $1.24 \pm 0.14$ & $1.31 \pm 0.34$ \\
\hline$a_{5}$-integrin & $3.57 \pm 0.52$ & $3.48 \pm 0.35$ & $7.30 \pm 0.60 *, \#$ & $3.00 \pm 0.30$ & $3.18 \pm 0.37$ & $5.68 \pm 0.34^{*, \#}$ \\
\hline$a_{v}$-integrin & $1.12 \pm 0.04$ & $1.01 \pm 0.18$ & $2.30 \pm 0.2^{*, \#}$ & $0.88 \pm 0.07$ & $0.98 \pm 0.12$ & $1.34 \pm 0.11^{*, \#}$ \\
\hline FAK & $1.62 \pm 0.23$ & $6.25 \pm 0.07 *$ & $7.39 \pm 0.09 *, \#$ & $1.60 \pm 0.10$ & $2.62 \pm 0.26^{*}$ & $4.25 \pm 0.07^{*, \#}$ \\
\hline MMP2 & $6.25 \pm 1.25$ & $6.74 \pm 0.81$ & $10.03 \pm 0.67 *, \#$ & $4.76 \pm 0.38$ & $5.14 \pm 0.46$ & $8.59 \pm 0.96^{*, \#}$ \\
\hline
\end{tabular}

Results are expressed in percentage of colored or stained media area and reported as the mean \pm SEM. $n=6-8$ rats per group. MCSA, media cross-sectional area; BW, body weight; FAK, focal adhesion kinase; MMP2, matrix metalloproteinase- $2 .{ }^{*}$ Significantly different $(p<0.05)$ compared to WKY; ${ }^{*}$ significantly different $(p<0.05)$ compared to SHRSP.

was a further significant increase in medial thickness and MCSA following salt administration. In agreement with in vivo echotracking measures, the diameter was increased in the thoracic but not the abdominal aorta. Also, the ratio thickness/lumen diameter was increased only at the abdominal aortic site. These measurements were also performed at an upper thoracic site to determine the viability of our chosen section of thoracic aorta to represent the whole thoracic aorta: thickness was increased from $109 \pm 9$ to $136 \pm 5$ and $148 \pm 3 \mu \mathrm{m}$ in the WKY, SHRSP, and SHRSP-salt groups, respectively. However, the thickness/lumen ratio was not different in these respective groups: $7.7 \pm 0.6,8.1 \pm 0.1$, and $8.5 \pm 0.2$. Since this section exhibited similar changes to that of our chosen lower thoracic site (between diaphragm and renal arteries), we considered our thoracic site to be representative.

Wall stress values calculated for MBP after clonidine administration were higher in the thoracic aorta than in the abdominal aorta in the 3 groups: $895 \pm 20$ versus 615 \pm 31 in WKY; $825 \pm 32$ versus $477 \pm 17$ in SHRSP; $753 \pm$ 23 versus $480 \pm 21$ in SHRSP-salt. Wall stress was similar in the SHRSP and SHRSP-salt groups at the abdominal site. Since MBP was also the same between these 2 groups, this provides greater evidence that the reductions in distensibility of the SHRSP-salt rats reflects a true change in the structure of the arterial wall. Wall stress was highly correlated to thickness/internal diameter ratio as expected $(r=0.91$ and 0.97$)$, slightly correlated to $\mathrm{MBP}(r=0.55$ and 0.47 in thoracic and abdominal aorta), and not correlated to stiffness ( $r=0.46$ and 0.44 ).

The vascular collagen content and density remained unchanged at both aortic levels for each group, irrespective of the salt loading (Table 3). The organization of elastic material in the thoracic wall appeared parallelly oriented with only a few disruptions of elastic lamellae for both WKY and SHRSP (Fig. 4). While the number of SHRSP-salt elastic lamellae was maintained at the thoracic site, they often appeared thinner than in the control groups, leading to significantly less elastin content (Table 3).

Thinner elastic lamellae and sparser interlamellar elastic fibers were observed in the abdominal aorta, such that 


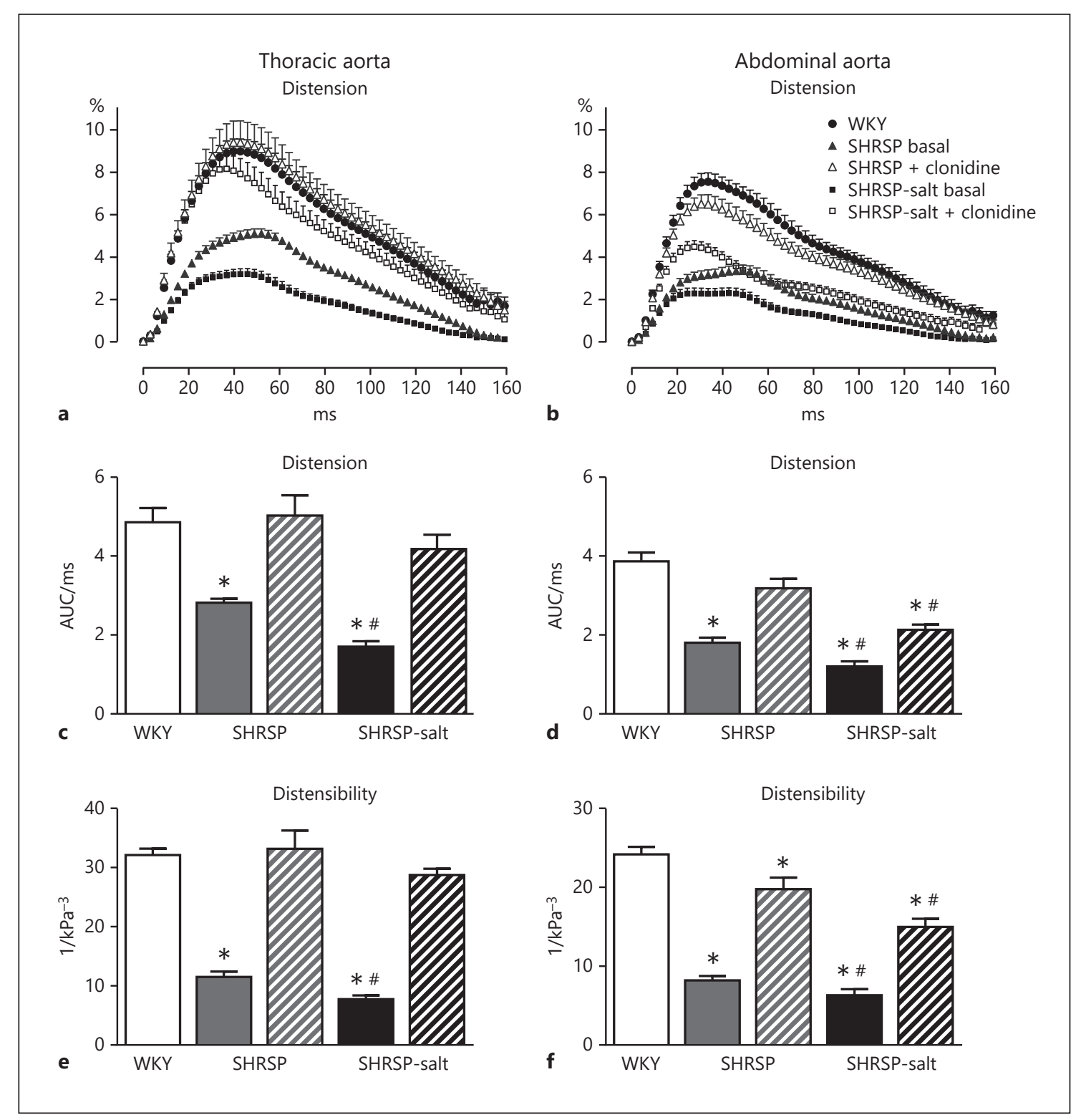

Fig. 2. Comparison of the differences in thoracic and abdominal stiffness as represented by changes in AUC of aortic distension throughout the cardiac cycle. The thoracic aorta demonstrates a higher intrinsic distensibility compared to the abdominal aorta ( $\mathbf{a}, \mathbf{c}, \mathbf{e}$ vs. $\mathbf{b}, \mathbf{d}, \mathbf{f})$. For images $\mathbf{c}-\mathbf{f}$, the unstriped bars represent the values at baseline BPs for each group whereas the striped bars represent the match BPs to the WKY. ${ }^{*} p<0.05$ for comparisons with WKY; ${ }^{p} p<0.05$ for comparisons with SHRSP.

elastin density was decreased compared with the thoracic site for each group. This result likely contributes to the greater stiffness observed in the abdominal compared with the thoracic aorta. The elastin network also showed varying degrees of disorganization between each group at the abdominal site. A loss of parallel orientation of elastin fibers was observed for all groups at the abdominal compared with the thoracic level (Fig. 4).
Increased elastin disarray (dispersion and rupture of elastic fibers) was increased at the abdominal aorta level after salt loading compared with both controls (2.86 \pm 0.06 for SHRSP-salt vs. $2.36 \pm 0.08$ for SHRSP and $1.80 \pm$ 0.15 for WKY; $p<0.001$ ). This tissue specificity of elastin content does not seem to be related to MMP2 accumulation as the latter increases to the same extent in both the thoracic and abdominal aorta (Table 3). The density of 


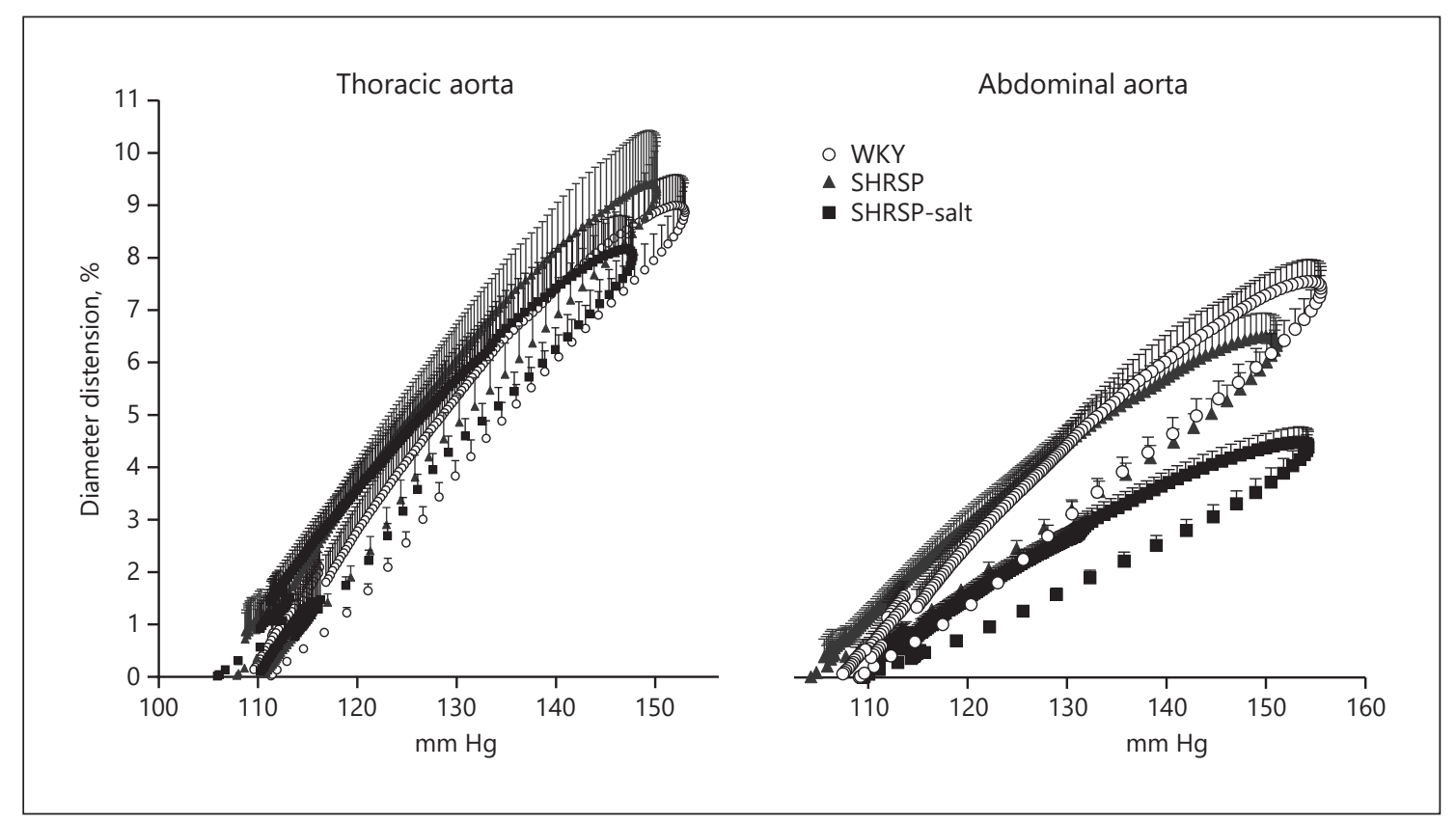

Fig. 3. Pressure-distension loop comparisons between the thoracic aorta and the abdominal aorta of all 3 groups at a common BP. It is clear to see that while the SHRSP at both sites and the SHRSP-salt at the thoracic site return to a normal pressure-distension loop defined by the WKY, the SHRSP-salt in the abdominal aorta remains significantly different.

cell nuclei was increased at the thoracic and abdominal aorta levels for SHRSP and SHRSP-salt compared with WKY. Concerning cell-matrix interactions, we observed that fibronectin content was specifically increased in SHRSP-salt rats compared to WKY and SHRSP rats at both aortic sites (Fig. 5, 6). This accumulation of fibronectin was coupled to a specific increase of its $\alpha_{5^{-}}$and $\alpha_{v^{-}}$ integrin receptors. FAK accumulation, which recognizes FAK, was consistent with integrin engagement in SHRSPsalt rats, even if a smaller increase of FAK accumulation was also observed in SHRSP rats compared to WKY.

\section{Discussion}

The major findings of this investigation are that: (1) increased dietary salt induced aortic stiffening in a saltsensitive model of hypertension, and (2) the thoracic and abdominal aorta exhibit different patterns of stiffening associated with different structural changes in the vessel wall and this is exacerbated after salt loading. Notably, salt induced a pressure-independent stiffness increase in the abdominal aorta which was more specifically related to elastin network alterations.

Salt-Induced Aortic Stiffening in Rats
The search for animal models which present with increased arterial stiffness is of great importance to characterize and understand the contribution of various factors associated with this cardiovascular risk factor in humans. The SHRSP is characterized by even higher BPs than SHR, a tendency to die from stroke, and a sensitivity to salt. The SHRSP presents with an increase in oxidative stress, a reduction in nitric oxide availability, and endothelial dysfunction $[12,13,21,25]$. A decrease in nitric oxide bioavailability has been previously shown to induce strong aortic stiffening $[17,26]$. Furthermore, since the SHRSP rats under a salted diet also present renal dysfunction [14] and renal dysfunction is commonly associated with increases in aortic stiffness [16,27], we hypothesized that salt-sensitive SHRSP rats mimic hypertensive saltsensitive humans and would therefore present with increased arterial stiffness. The severity of the SHRSP model used in this study is confirmed by the clear cardiac and renal hypertrophy in SHRSP, which was further increased following chronic salt consumption.

SHRSP and SHRSP-salt animals exhibited decreased distensibility and aortic distension and an increased stiffness index and local PWV when compared to WKY rats, as measured by the vascular internal diameter (ultrasonic 
echotracking) coupled with simultaneous aortic BP measurements. This stiffening was visible at the thoracic and the abdominal level in SHRSP versus WKY rats. These results are in line with those published by Chapleau et al. [28]. Despite using a very different protocol which did not compare the SHRSP-salt group with a control group, they nicely describe the development over 4 weeks of treatment of salt-induced aortic stiffening measured both systemically via PWV and locally at the ascending aortic site.

The increase in BP due to chronic salt consumption in SHRSP $[12,14]$ was slight under anesthesia and made the results difficult to interpret at operational BP. Thus, analysis after BP adjustment to match WKY MBP was essential to compare thoracic and abdominal features and to distinguish pressure-dependent and independent stiffening as previously demonstrated [17].
At matched BP, the thoracic and abdominal aortic stiffness parameters in SHRSP were no longer different from those of WKY, except for abdominal distensibility, which was still decreased. These results indicate that SHRSP aortic stiffening was totally BP-dependent at the thoracic site but slightly altered in the abdominal site (Table 1; Fig. 2). Here we again highlight the importance of comparing arterial stiffness values at matched MBP [5, 17].

We used clonidine to lower $\mathrm{BP}$ via a reduction in total sympathetic output. In previous studies we used a nitric oxide donor but found that its effect on BP varies greatly in pathological models. In a model of severe aortic stiffening, we obtained similar results on stiffness and distensibility parameters upon administration of clonidine or a calcium antagonist. Any means of decreasing BP will af-

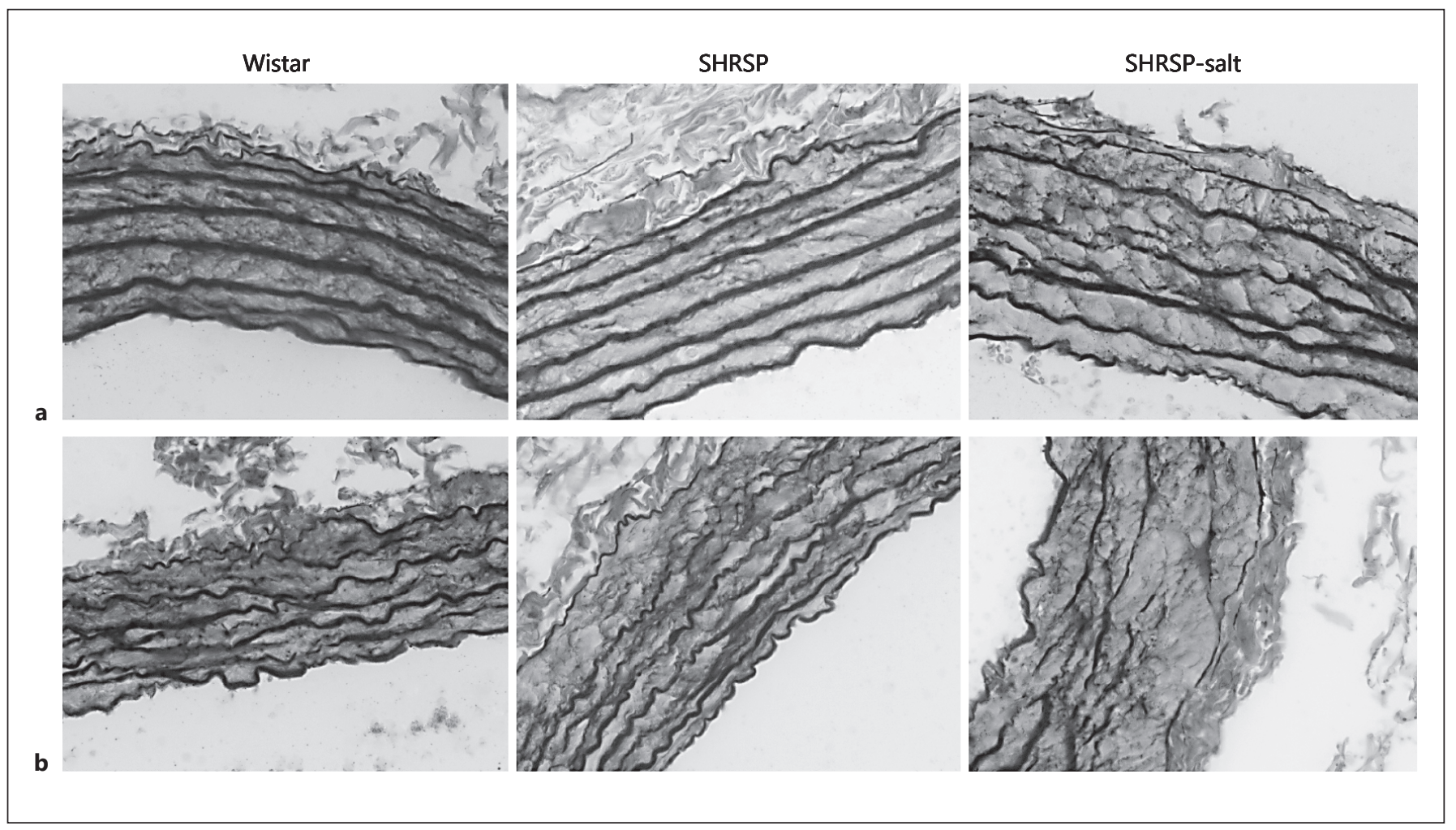

Fig. 4. Elastic fiber networks in transverse sections after Verhoeff's van Gieson staining of thoracic (a) and abdominal aortas (b) in WKY, SHRSP, and SHRSP-salt rats. The increased disarray of elastin networks in the medial layer, determined by loss of parallel orientation and increased dispersion of fibers, was obvious between thoracic and the abdominal sites for each group. In SHRSP-salt, elastin disarray was also increased compared with both WKY and SHRSP at the abdominal site.

Fig. 5. Representative images of fibronectin, its $\alpha_{5}$-integrin receptor, and FAK of the thoracic aorta in WKY, SHRSP, and SHRSP-salt rats. Collagen is also presented in blue after Masson's trichrome coloration.

(For figure see next page.) 


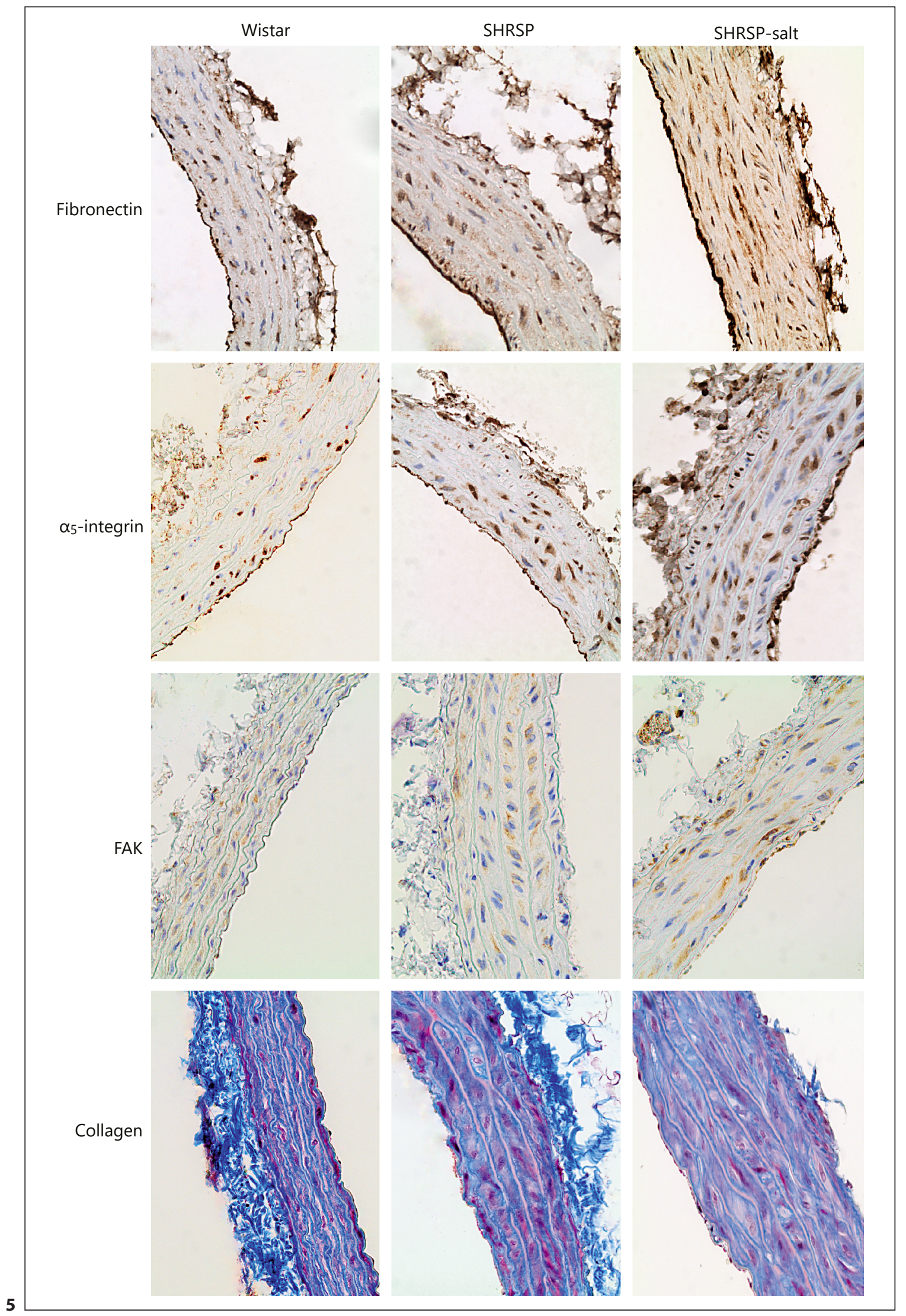




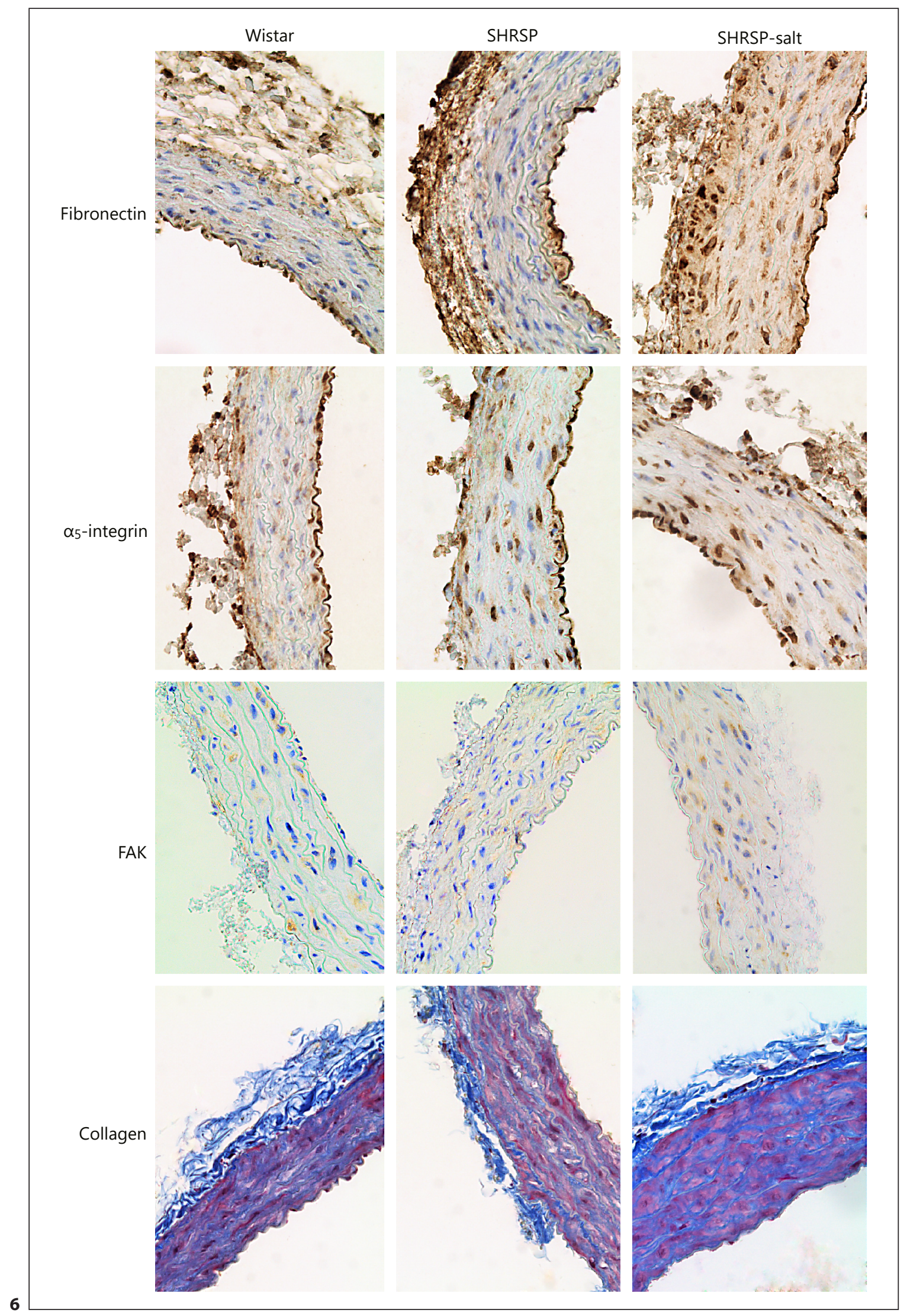

(For legend see next page.) 
fect vascular tone, and clonidine was chosen because of its long action and its ability to be titrated to achieve a precise decreases in BP. At matched BP levels, the differential effects of salt upon the thoracic and abdominal aorta were clearly revealed. In the abdominal aorta, distensibility and distension remained decreased and stiffness index and local PWV increased in the SHRSP-salt compared to both the WKY and SHRSP animals, indicating the pressure-independent stiffening. To date, this is the first study which has shown a BP-independent increase in aortic stiffness in SHRSP-salt rats. The thoracic aorta did not present such BP-independent changes. This observation is the second major finding of this study, indicating that the addition of dietary salt produced a site-specific increase in arterial stiffness. The analysis of the pressure and distension waveforms confirms these observations, as well as the distension-pressure loop analysis shown in Figures 2 and 3, as was previously shown [17], and highlight these thoracic-abdominal differences.

It may have been more informative to match wall stress values than MBP values; however, this was not feasible with our recording instruments. In contrast to BP, which is continuously recorded and observed online, the recorded diameter values are measurable only after the experiment; moreover, in rats wall thickness is not detectable in vivo and this parameter is only measured after euthanasia on histological sections. Presently, there is no means of actively calculating wall stress in vivo and adjusting it accordingly in rats. Thus, BP values are related to histological fix values of diameter and thickness whatever the pressure and pulse pressure were at different periods of recording. However, it is important to note that the major interest and originality of our technique is to measure dynamic parameters, which take into account pulsatile changes in alive animals. Nevertheless, wall stress values were calculated offline at reduced pressure values. We observed that, as expected, wall stress was lower in the abdominal aorta than in the thoracic aorta. Secondly, we observed that wall stress was slightly lower in hypertensive rats than in normotensive rats, which could be viewed as a limitation of the study. Nonetheless, our technique of normalizing our measurements for both MBP and pulse pressure is the most viable option during experiments and allows the exploitation of much more data (i.e., distension curves and pressure-distension

Fig. 6. Representative images of the abdominal aorta stained for the same proteins (fibronectin, $\alpha_{5}$-integrin, FAK, and collagen) and groups of rats (WKY, SHRSP, and SHRSP-salt) used in the Figure 5.

Salt-Induced Aortic Stiffening in Rats loops). Concerning wall stress, it was therefore more relevant to compare the 2 groups of hypertensive rats; these results showed that wall stress was equal at the abdominal sites with and without salt. This again suggests that the salt-induced pressure independent increases in stiffness at this site were due to changes in the structure of the vessel wall and not changes in vessel caliber.

Our observations are consistent with data showing that the abdominal aorta is more prone to arteriosclerosis than the thoracic aorta $[29,30]$. The aorta is often considered as a single entity; however, arterial stiffening develops differently along the aortic bed with aging in both humans and animal models [31-33]. This may be due to the fact that the thoracic and abdominal aorta have different embryologic origins, different means of maturation, and different structural and mechanical properties $[34,35]$. Hickson et al. [31] showed that, in humans, aging resulted in a greater increase in abdominal aortic PWV compared to the thoracic aorta. Furthermore, Dodson et al. [32] were able to induce an increase in abdominal stiffness whilst having very little effect on the thoracic aorta in a model of sheep placental insufficiency. These changes were attributed to differential structural remodeling at each site. More recently, Zhang et al. [33] showed that the abdominal aorta is more severely stiffened with age in monkeys and that this was correlated more so with a disarray of collagen and elastin fibers than the traditional measure of the elastin/collagen ratio. The increased stiffness observed at the abdominal level, compared with the thoracic aorta, should be explained first by differences of the geometry of the artery (differential remodeling between the 2 sites) and by the composition of the aortic wall.

Concerning the geometry, we observed a progressive increase in wall thickness from the normotensive, to the SHRSP, to the SHRSP-salt at both the thoracic and abdominal sites. Nevertheless, relative to the internal aortic diameter, the abdominal aorta showed a greater and inward increase in aortic wall thickness with respect to the thoracic aorta. Increases in wall thickness are commonly seen as a result of chronic hypertension whereby the aortic media thickens to better distribute tension across the aortic wall [36]. Increases in wall thickness have also been shown to occur independently of changes in BP, notably in studies of salt consumption which lead to the proliferation of vascular smooth muscle cells and development of the extracellular matrix in the spontaneously hypersensitive rat $[37,38]$ without association with increased stiffness [39]. Our data demonstrate that if the thickening of the vascular wall is not necessarily associated with stiffening, inward remodeling is. 
A comparison of the aortic wall should also explain differences of stiffness between the 2 aortic sites. The sitespecific differences in aortic stiffness were derived from extracellular matrix changes in both aortic sites. Extracellular matrix proteins determine the passive biomechanical properties, collagen provides tensile strength, and elastin enables vascular elasticity [40]. A relationship between a decreased elastin/collagen ratio and arterial stiffness in different experimental models has long been described [41]. In our study, a lower elastin density was observed in the abdominal aorta compared with the thoracic aorta for each control strain (WKY and SHRSP), suggesting that it contributes to the greater stiffness observed in the abdominal site compared with the thoracic. Paradoxically, as already reported by Contri et al. [42], elastin density was specifically reduced at the thoracic level in the SHRSP-salt group compared with both controls, but not at the abdominal site. This indicates that other factors than solely elastin density may account for the abdominal stiffness increase in this model, and different mechanisms regulate elastin density at both levels. This observation is not entirely novel since conflicting trends in aortic scleroprotein contents have long been noted, indicating that other extracellular matrix proteins, crosslinking, glycation, and structural or architectural rearrangement may contribute to altering the mechanical properties of the vascular wall [43]. It was recently shown that scleroprotein disarray as opposed to elastin/collagen ratio changes accounted for the increase in abdominal aortic stiffness versus thoracic stiffness in aged primates [33]. In this study, the authors demonstrated that, as in our salt model, elastin disarrays were more pronounced at the abdominal site and thus may account for the observed increased in stiffness. Whilst elastin disarray appears associated with aortic stiffness, the current mechanism associated with these alterations is currently unknown. However, previous papers by Samokhin et al. [44] reported that cysteinyl cathepsins $(\mathrm{CstS} / \mathrm{K})$ are one of the key enzymes to degrade elastic lamina of the atherosclerotic plaques. Thus, future work should evaluate the levels of these cathepsins in models of aortic stiffness to fully determine their role in enhancing elastin disarray.

Other obvious structural changes of the aorta shown in the present study were an accumulation of fibronectin and its integrin receptors. This is typically associated with arterial stiffness and already known to be upregulated during hypertension and aging [19]. To better characterize cell-extracellular matrix interactions, we also investigated focal adhesions through FAK quantification. Focal adhesions are becoming more widely recognized as me- diators of arterial stiffness since they are responsible for the connections made between the extracellular matrix and vascular small muscle cells [45]. We can only speculate how the identical increased expression of focal adhesion markers seen in both the abdominal aorta and thoracic aorta can lead to an increase in stiffness in the former and not the latter. Altogether, these results provide evidence that, in SHRSP, salt induces a profound remodeling and fibrosis which is strongly correlated with differences in the pressure-independent stiffening between thoracic and abdominal aorta.

The thoracic section that we evaluated between the diaphragm and renal bifurcation is short and much less studied than the portion above the diaphragm, in vivo as well as in vitro. However, in echotracking experiments it presents the advantage of being recorded without opening the thoracic cage and by good vascular landmarks and thus reproducibility. Yet the reason why we chose to evaluate this level as thoracic was not only driven by technical considerations. Even though the diaphragm or the renal arteries are alternatively defined as the limit between the thoracic and abdominal aorta in the literature, we hypothesized that the renal bifurcation was the real transition point of embryological differentiation because the diaphragm has no physiological relationship with the aorta whilst the renal arteries certainly do. We nevertheless aimed to test this hypothesis by comparing thickness and diameter measurements at the suprarenal site and also above the diaphragm. We showed that the thickness/ lumen ratio of these 2 aortic sites were equal and significantly different from the abdominal site, indicating they were of the same embryological origin. Moreover, we observed that our thoracic site had more medial elastin layers than the abdominal aorta [34], confirming the thoracic origin of the site between the diaphragm and renal arteries.

According to our results, future research should consider techniques such as the evaluation of the properties and waviness of collagen fibers, which also account for the dynamic properties of the vascular wall [46] and scanning acoustic microscopy [47] to determine how and where the observed changes in the extracellular matrix translate to increases in local arterial stiffness. In further studies it may also be interesting to investigate whether gender contributes to salt-induced changes in aortic stiffness.

The results of the study suggest that the thoracic aorta, which has an important role in dampening cardiac output, appears less sensitive to salt-induced stiffening, thus limiting changes to the left ventricle. One limitation of the 
study is that this observation was made over a restricted period of time. These differences need to be evaluated both in other pathological conditions and also after a longer treatment period to evaluate the possible development of pressure-independent stiffening of the thoracic aorta over time. Another limitation of such studies is that echotracking measurements in animals requires anesthesia. Nonetheless, this technique allows in vivo recordings of local arterial wall stiffness; it provides valuable information on the functional deleterious effects of salt, thus allowing us to advance our understanding on the pathological consequences of salt in hypertensive humans.

In conclusion, we have demonstrated that the addition of dietary salt to the salt-sensitive SHRSP induces local- ized changes in aortic stiffness. These changes are associated with morphohistochemical modifications, and reflect the different embryological origins, suggesting functional differences between the thoracic and abdominal aorta. These findings highlight the importance of experimental models to fully investigate local aortic stiffness in disease conditions with the aim to screen and develop specific pharmacologic targets.

\section{Disclosure Statement}

The authors have no conflicts of interest

\section{References}

$\checkmark 1$ Meaume S, Benetos A, Henry OF, Rudnichi A, Safar ME: Aortic pulse wave velocity predicts cardiovascular mortality in subjects $>70$ years of age. Arterioscler Thromb Vasc Biol 2001;21:2046-2050.

-2 Laurent S, Boutouyrie P, Asmar R, Gautier I, Laloux B, Guize L, et al: Aortic stiffness is an independent predictor of all-cause and cardiovascular mortality in hypertensive patients. Hypertension 2001;37:1236-1241.

-3 Cruickshank K, Riste L, Anderson SG, Wright JS, Dunn G, Gosling RG: Aortic pulse-wave velocity and its relationship to mortality in diabetes and glucose intolerance: an integrated index of vascular function? Circulation 2002;106:2085-2090.

4 O'Rourke MF, Hashimoto J: Mechanical factors in arterial aging: a clinical perspective. J Am Coll Cardiol 2007;50:1-13.

5 Lindesay G, Ragonnet C, Chimenti S, Villeneuve N, Vayssettes-Courchay C: Age and hypertension strongly induce aortic stiffening in rats at basal and matched blood pressure levels. Physiol Rep 2016;4:e12805.

6 Celik T, Iyisoy A, Kursaklioglu H, Turhan H, Cagdas Yuksel U, Kilic S, et al: Impaired aortic elastic properties in young patients with prehypertension. Blood Press Monit 2006;11: 251-255.

7 Kaess BM, Rong J, Larson MG, Hamburg NM, Vita JA, Levy D, et al: Aortic stiffness, blood pressure progression, and incident hypertension. JAMA 2012;308:875-881.

$>8$ Avolio AP, Clyde KM, Beard TC, Cooke HM, Ho KK, O’Rourke MF: Improved arterial distensibility in normotensive subjects on a low salt diet. Arterioscler Dallas Tex 1986;6:166169.

$>9$ Safar ME, Thuilliez C, Richard V, Benetos A: Pressure-independent contribution of sodium to large artery structure and function in hypertension. Cardiovasc Res 2000;46:269276.

10 Herrera VL, Decano JL, Giordano N, Moran AM, Ruiz-Opazo N: Aortic and carotid arterial stiffness and epigenetic regulator gene expression changes precede blood pressure rise in stroke-prone Dahl salt-sensitive hypertensive rats. PLoS One 2014;9:e107888.

11 Kim-Mitsuyama S, Yamamoto E, Tanaka T, Zhan Y, Izumi Y, Izumiya Y, et al: Critical role of angiotensin II in excess salt-induced brain oxidative stress of stroke-prone spontaneously hypertensive rats. Stroke 2005;36:10831088 .

12 Zhang W, Liu A-J, Yi-Ming W, Liu J-G, Shen F-M, Su D-F: Pressor and non-pressor effects of sodium loading on stroke in stroke-prone spontaneously hypertensive rats. Clin Exp Pharmacol Physiol 2008;35:83-88.

13 Papazzo A, Conlan XA, Lexis L, Charchar FJ, Lewandowski PA: Salt loading in canola oil fed SHRSP rats induces endothelial dysfunction. PLoS One 2013;8:e66655.

14 Griffin KA, Churchill PC, Picken M, Webb RC, Kurtz TW, Bidani AK: Differential saltsensitivity in the pathogenesis of renal damage in SHR and stroke prone SHR. Am J Hypertens 2001;14:311-320.

15 Vanhoutte PM, Shimokawa H, Feletou M, Tang EHC: Endothelial dysfunction and vascular disease - a 30th anniversary update. Acta Physiol 2017;219:22-96.

16 Sedaghat S, Dawkins Arce FG, Verwoert GC, Hofman A, Ikram MA, Franco OH, et al: Association of renal function with vascular stiffness in older adults: the Rotterdam study. Age Ageing 2014;43:827-833.

17 Vayssettes-Courchay C, Ragonnet C, Isabelle M, Verbeuren TJ: Aortic stiffness in vivo in hypertensive rat via echo-tracking: analysis of the pulsatile distension waveform. Am J
Physiol Heart Circ Physiol 2011;301:H382H390.

18 Bouissou-Schurtz C, Lindesay G, Regnault V, Renet S, Safar ME, Molinie V, et al: Development of an experimental model to study the relationship between day-to-day variability in blood pressure and aortic stiffness. Front Physiol 2015;6:368.

19 Isabelle M, Chimenti S, Beaussier H, Gransagne D, Villeneuve N, Safar ME, et al: SBP, $\mathrm{DBP}$, and pulse blood pressure variability are temporally associated with the increase in pulse wave velocity in a model of aortic stiffness. J Hypertens 2016;34:666-675.

20 Brands PJ, Hoeks AP, Willigers J, Willekes C, Reneman RS: An integrated system for the non-invasive assessment of vessel wall and hemodynamic properties of large arteries by means of ultrasound. Eur J Ultrasound Off J Eur Fed Soc Ultrasound Med Biol 1999;9: 257-266.

21 Endemann DH, Touyz RM, Iglarz M, Savoia C, Schiffrin EL: Eplerenone prevents saltinduced vascular remodeling and cardiac fibrosis in stroke-prone spontaneously hypertensive rats. Hypertension 2004; 43: 1252-1257.

-22 Sukhova GK, Zhang Y, Pan J-H, Wada Y, Yamamoto T, Naito M, et al: Deficiency of cathepsin $\mathrm{S}$ reduces atherosclerosis in LDL receptor-deficient mice. J Clin Invest 2003;111: 897-906.

23 Bezie Y, Lacolley P, Laurent S, Gabella G: Connection of smooth muscle cells to elastic lamellae in aorta of spontaneously hypertensive rats. Hypertension 1998;32:166-169.

24 Bézie Y, Lamazière JM, Laurent S, Challande $\mathrm{P}$, Cunha RS, Bonnet J, et al: Fibronectin expression and aortic wall elastic modulus in spontaneously hypertensive rats. Arterioscler Thromb Vasc Biol 1998;18:1027-1034. 
-25 Takemori K, Inoue T, Ito H: Possible role of nitric oxide generated by leukocytes in the pathogenesis of hypertensive cerebral edema in stroke-prone spontaneously hypertensive rats. Brain Res 2011;1417:137-145.

26 Isabelle M, Simonet S, Ragonnet C, Sansilvestri-Morel P, Clavreul N, Vayssettes-Courchay $\mathrm{C}$, et al: Chronic reduction of nitric oxide level in adult spontaneously hypertensive rats induces aortic stiffness similar to old spontaneously hypertensive rats. J Vasc Res 2012;49: 309-318.

27 Guerin AP, Blacher J, Pannier B, Marchais SJ, Safar ME, London GM: Impact of aortic stiffness attenuation on survival of patients in end-stage renal failure. Circulation 2001;103: 987-992.

28 Chapleau MW, Rotella DL, Reho JJ, Rahmouni K, Stauss HM: Chronic vagal nerve stimulation prevents high-salt diet-induced endothelial dysfunction and aortic stiffening in stroke-prone spontaneously hypertensive rats. Am J Physiol Heart Circ Physiol 2016; 311:H276-H285.

29 Roberts JC, Wilkins RH, Moses C: Autopsy studies in atherosclerosis. Circulation 1959; 20:520-526.

- 30 Wolinsky H, Glagov S: A lamellar unit of aortic medial structure and function in mammals. Circ Res 1967;20:99-111.

- 31 Hickson SS, Butlin M, Graves M, Taviani V, Avolio AP, McEniery CM, et al: The relationship of age with regional aortic stiffness and diameter. JACC Cardiovasc Imaging 2010;3: 1247-1255.
32 Dodson RB, Rozance PJ, Petrash CC, Hunter KS, Ferguson VL: Thoracic and abdominal aortas stiffen through unique extracellular matrix changes in intrauterine growth restricted fetal sheep. Am J Physiol Heart Circ Physiol 2014;306:H429-H437.

33 Zhang J, Zhao X, Vatner DE, McNulty T, Bishop S, Sun Z, et al: Extracellular matrix disarray as a mechanism for greater abdominal versus thoracic aortic stiffness with aging in primates. Arterioscler Thromb Vasc Biol 2016;36:700-706.

34 Wolinsky H: Comparison of medial growth of human thoracic and abdominal aortas. Circ Res 1970;27:531-538.

35 Ruddy JM, Jones JA, Spinale FG, Ikonomidis JS: Regional heterogeneity within the aorta: relevance to aneurysm disease. J Thorac Cardiovasc Surg 2008;136:1123-1130.

36 Wolinsky H: Long-term effects of hypertension on the rat aortic wall and their relation to concurrent aging changes: morphological and chemical studies. Circ Res 1972;30:301309.

37 Limas C, Westrum B, Limas CJ, Cohn JN: Effect of salt on the vascular lesions of spontaneously hypertensive rats. Hypertension 1980;2: 477-489.

38 Partovian C, Benetos A, Pommiès JP, Mischler W, Safar ME: Effects of a chronic highsalt diet on large artery structure: role of endogenous bradykinin. Am J Physiol 1998; 274:H1423-H1428.

39 Laurent S, Boutouyrie P, Lacolley P: Structural and genetic bases of arterial stiffness. Hypertension 2005;45:1050-1055.

40 Wolinsky H, Glagov S: Comparison of abdominal and thoracic aortic medial structure in mammals: deviation of man from the usual pattern. Circ Res 1969;25:677-686.
41 Lacolley P, Thornton SN, Bezie Y: Animal models for studies of arterial stiffness; in Safar ME, O'Rourke MF, Frohlich ED (eds): Blood Pressure and Arterial Wall Mechanics in Cardiovascular Diseases. London, Springer, 2014, pp 63-74

42 Contri MB, Taparelli F, Miselli M, Bacchelli B, Biagini G: Histomorphometric, biochemical and ultrastructural changes in the aorta of salt-loaded stroke-prone spontaneously hypertensive rats fed a Japanese-style diet. Nutr Metab Cardiovasc Dis 2003;13:37-45.

43 Sehgel NL, Vatner SF, Meininger GA: "Smooth muscle cell stiffness syndrome" - revisiting the structural basis of arterial stiffness. Front Physiol 2015;6:335.

44 Samokhin AO, Wong A, Saftig P, Brömme D: Role of cathepsin $\mathrm{K}$ in structural changes in brachiocephalic artery during progression of atherosclerosis in apoE-deficient mice. Atherosclerosis 2008;200:58-68

45 Saphirstein RJ, Gao YZ, Jensen MH, Gallant CM, Vetterkind S, Moore JR, et al: The focal adhesion: a regulated component of aortic stiffness. PLoS One 2013;8:e62461.

46 Schrauwen JTC, Vilanova A, Rezakhaniha R, Stergiopulos N, van de Vosse FN, Bovendeerd PHM: A method for the quantification of the pressure dependent 3D collagen configuration in the arterial adventitia. J Struct Biol 2012;180:335-342.

47 Akhtar R, Cruickshank JK, Zhao X, Derby B, Weber T: A pilot study of scanning acoustic microscopy as a tool for measuring arterial stiffness in aortic biopsies. Artery Res 2016; 13:1-5. 